\title{
Disfunción sexual femenina: una mirada a los últimos 44 años. Análisis bibliométrico
}

\section{Female Sexual Dysfunction: Looking Back at the Last 44 Years. A Bibliometric Analysis}

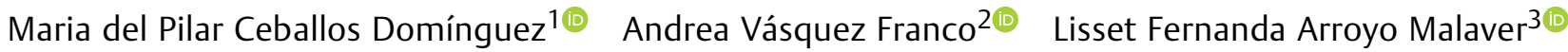 \\ Luisana Castillo Carvajal4,5® Johanna María Montes ${ }^{6 \odot}$ Pamela Perdomo $^{7(}$ Johanna Vega ${ }^{8(0)}$ \\ Catalina Solano Mendoza20
}

\footnotetext{
1 Departamento de Urología, Colsanitas, Medicina Sexual, Bogotá, Colombia

2 Departamento de Urología, Uroclin, Bogotá, Colombia

${ }^{3}$ Departamento de Urología, Hospital Simón Bolívar, Bogotá, Colombia

${ }^{4}$ Departamento de Urología, Clínica Santa María del Lago, Bogotá, Colombia

${ }^{5}$ Departamento de Urología, Clínica Juan N. Corpas, Bogotá, Colombia 6 Departamento de Urología, Clínica Primavera, Villavicencio, Colombia

7 Departamento de Urología, Clínica Antioquia, Antioquia, Colombia

${ }^{8}$ Departamento de Urología, Hospital Universitario de la Samaritana, Bogotá, Colombia
}

Urol Colomb 2021;30(4):e304-e312.
Dirección para correspondencia María del Pilar Ceballos Domínguez, Médico Urólogo, Medicina Sexual Calle 127 Bis \# 19 -25, Consultorio 401, Edificio 127, Bogotá, Colombia (e-mail: pilarceballosurologa@yahoo.com).

\section{Resumen \\ Palabras Clave \\ - disfunción sexual femenina \\ - sexualidad \\ - femenina \\ - urología \\ - bibliométrico \\ - salud sexual y reproductiva}

Objetivo La disfunción sexual femenina (DSF) es un trastorno prevalente que afecta la calidad de vida de las mujeres y combina factores biológicos, psicológicos e interpersonales. Su estudio ha sido de poco interés, en campos como la investigación y el tratamiento, debido a la dificultad en la medición de las respuestas sexuales en las mujeres y otros factores, como el tabú acerca de la sexualidad femenina. Este estudio caracteriza las tendencias de publicaciones científicas acerca del tema, para que los urólogos puedan comprender la necesidad de realizar la búsqueda de este trastorno en la práctica diaria.

Métodos Se realizó una búsqueda de la literatura en las bases de datos PubMed, FABUMED y Scopus, utilizando la estrategia "((«female sexual dysfunction» [Title/Abstract])». Se realizó un análisis bibliométrico descriptivo, de corte retrospectivo, de la literatura médica obtenida en MEDLINE. A través de las herramientas estadísticas FABUMED, PubReminer y Scopus, se recopilaron los datos y se obtuvo los resultados de las variables que posteriormente fueron analizadas de forma estadística y descriptiva. Con base en esta información, elaboramos tablas y gráficas en Microsoft Office Excel 2017. Adicionalmente, realizamos un análisis de mapeo bibliométrico utilizando el programa VOSviewer. Para la obtención del factor de impacto (FI), utilizamos el Journal Citation Reports 2017/2018. recibido

02 de septiembre de 2020

aceptado

16 de mes de julio de 2021
DOI https://doi.org/ $10.1055 / \mathrm{s}-0041-1740344$. ISSN 0120-789X.

e ISSN 2027-0119. (c) 2021. Sociedad Colombiana de Urología. All rights reserved. This is an open access article published by Thieme under the terms of the Creative Commons Attribution-NonDerivative-NonCommercial-License, permitting copying and reproduction so long as the original work is given appropriate credit. Contents may not be used for commercial purposes, or adapted, remixed, transformed or built upon. (https://creativecommons.org/ licenses/by-nc-nd/4.0/)

Thieme Revinter Publicações Ltda., Rua do Matoso 170, Rio de Janeiro, RJ, CEP 20270-135, Brazil 
Resultados Desde 1975 hasta 2019, se publicaron 1.292 estudios, y la tasa anual de publicación permaneció estable entre 1975 y 2000. Pero, a partir del año 2000, se evidenció un aumento en el número de publicaciones, con un crecimiento del 92,1\%. De las veinte revistas con más publicaciones, sólo cuatro son de urología. Los países con más publicaciones fueron: Estados Unidos (31,4\%), Reino Unido (12,9\%), Italia (8,9\%), Turquía (5,9\%), y Australia (4\%). Estos datos son muy diferentes a los de los países de América Latina, en los que se encontraron muy pocas publicaciones, como Colombia, con tan sólo tres estudios.

Conclusiones Este análisis bibliométrico mostró las diferentes tendencias y tasas de publicación de estudios sobre DSF, y evidenció una baja tasa de producción con relación a otras temáticas, sobre todo en las revistas urológicas, con predominio de mujeres como autores, aunque en los últimos años se ha identificado un crecimiento importante y sostenido. Se hace evidente la necesidad de desarrollar estrategias para mejorar la formación tanto en los niveles de posgrado como de pregrado y, así, generar más adhesión a este tema en una especialidad quirúrgica como la nuestra.

Abstract

\section{Keywords}

- female sexual dysfunction

- sexuality

- female

- urology

- bibliometric

- sexual and reproductive Health
Objectives Female sexual dysfunction (FSD) is a prevalent disorder that affects the quality of life of women and combines biological, psychological, and interpersonal factors. There has been a lack of interest in studying FSD in such fields as research and treatment, due to the difficulty in measuring the sexual responses of women and other factors such as the taboo surrounding female sexuality. The present study portrays the trends in the scientific publications on the subject, so urologists can perceive and understand the need for research about this disorder in the daily practice.

Methods A comprehensive review on the topic was performed through a PubMed, FABUMED, and Scopus databases using the search strategy "female sexual dysfunction", as well as a retrospective, descriptive, bibliometric analysis of the medical literature retrieved from MEDLINE. Data was collected through the FABUMED, PubReMiner, and Scopus statistical tools, and, based on the results obtained for the variables, which were later submitted to a statistical and descriptive analysis. Based on this data, tables and graphs were developed using the Microsoft Office Excel 2017 software. Moreover, we performed a bibliometric mapping analysis using the VOSviewer software. In order to obtain the impact factor (IF), we used the Journal Citation Reports 2017/2018.

Results From 1975 to 2019, 1,292 studies were published; yearly publication rate remained stable from 1975 to 2000 , but after year 2000, an increase in the number of publications was evidenced, with a growth of $92.1 \%$. But as of the year 2000 , an increase in the number of publications was evidenced, with a growth of $92.1 \%$. Of the twenty journals with the most publications, only four are on urology. The countries with the greatest number of publications were: the United States (31.4\%), the United Kingdom (12.9\%), Italy (8.9\%), Turkey (5.9\%), and Australia (4\%). This data differs from that of Latin American countries, in which few publications were found, such as in Colombia, with only three studies.

Conclusions The present bibliometric analysis showed the different trends and publication rates of studies on FSD, showing a low production rate as compared with that of other urological topics, especially in Urological journals, with a predominance of women as authors, although in recent years an important and sustained growth has been identified. The need to develop strategies to improve training at both the graduate and undergraduate levels and thus generate more adherence to these issues in a surgical specialty like ours is evident. 


\section{Introducción}

La disfunción sexual femenina (DSF) es la alteración en la salud sexual de las mujeres a causa de uno o varios factores biológicos, hormonales o psicológicos; y, al intervenir diferentes componentes, como la edad de las pacientes, factores sociales o estrés, generan como resultado un gran impacto en la calidad de vida. ${ }^{1-3}$ Según datos epidemiológicos, es un trastorno muy prevalente, que afecta entre un $20 \%$ y un $50 \%$ de las mujeres, y se presenta en todas o en alguna de las fases del ciclo sexual femenino. Un tercio de las pacientes carece de interés sexual o anorgasmia, el 20\% informa dificultades de lubricación, y el 20\% restante considera que el sexo no es placentero. ${ }^{4}$

A pesar de esta problemática, la clasificación y las causas de la DSF se han abordado principalmente en el la quinta edición del Manual diagnóstico y estadístico de los trastornos mentales (Diagnostic and Statistical Manual of Mental Disorders, fifth, edition, DSM-5, en inglés), ${ }^{5}$ pero no se ha explorado ampliamente en otros escenarios académicos. Por lo tanto, hay una falta de consenso en relación con la clasificación y los criterios diagnósticos para la DSF, y no queda claro cuáles especialidades están llamadas a liderar el manejo de esta condición ni el enfoque ideal que deberían tener las pacientes. ${ }^{1,6,7}$

Desde el punto de vista urológico, su abordaje no ha despertado tanto interés en el campo de la investigación y tratamiento, pues las respuestas sexuales femeninas han resultado mucho más difíciles de cuantificar, en contraste a la disfunción sexual masculina, en la que los estudios se han centrado principalmente en las erecciones y su manejo farmacológico. 8,9

Este tipo de estudio, conocido como análisis bibliométrico, permite evaluar y caracterizar de forma objetiva la importancia, la calidad y el impacto de los artículos científicos y, a su vez, la comparación académica entre investigadores, especialidades y revistas, buscando, en este caso, que los urólogos tengan una mejor comprensión de la necesidad de afrontar este tema en la práctica diaria, y que estén a la par de los abordajes actuales para los hombres con disfunción sexual. ${ }^{10}$ Por lo anterior, el objetivo del presente trabajo es revisar las tendencias de publicación de DSF en los últimos años para brindar una aproximación a las publicaciones sobre el tema en el campo de la urología en contraste con otras especialidades.

\section{Métodos}

Realizamos un análisis bibliométrico descriptivo, de corte retrospectivo. Para la búsqueda, utilizamos las bases de datos PubMed, FABUMED y Scopus, utilizando la estrategia "((«female sexual dysfunction» [Title/Abstract]) AND (1975:2019[dp]))», sin restricción en el idioma. Se incluyeron artículos originales de investigación, artículos de revista, ensayos, informes científicos y reseñas, con el fin de analizar toda la literatura médica indexada en relación con este tema.
Para la recopilación de datos, utilizamos las herramientas estadísticas FABUMED, PubReMiner y Scopus, y obtuvimos los resultados de las variables: número total de publicaciones y revistas; número y porcentaje de publicaciones por revista y país; patrón de producción científica a lo largo del período estudiado; autores; y artículos más citados. Adicionalmente, realizamos un análisis de mapeo bibliométrico utilizando técnicas de agrupamiento en el programa VOSviewer (Centre for Science and Technology Studies, Leiden University, The Netherlands), que explora las interacciones de palabras clave y títulos y resúmenes de la literatura encontrada, con el fin de mapear el dominio del conocimiento científico y revelarle al lector una manera simple y rápida de ver los datos.

Para la obtención del factor de impacto (FI), utilizamos el Journal Citation Reports 2017/2018 de las revistas encontradas, para estimar la calidad de cada una de estas. Adicionalmente, recuperamos la mediana de citas por artículo (MCA) y la mediana de citas por revisión (MCR) del Journal Citation Reports de las revistas específicas de urología. A diferencia del FI de la revista, la mediana de cita por artículo o revisión es una representación más útil del número total de citas que se espera recibir de un artículo cuando se publica en una revista específica.

Posterior a esto, analizamos los resultados de forma estadística y descriptiva y, con base en esta información, elaboramos tablas y gráficas en el programa Microsoft Office Excel 2017 (Microsoft Corp., Redmond, WA, EEUU).

\section{Resultados}

\section{Datos Generales}

Se publicaron un total de 1.292 estudios en 425 revistas desde 1975 a 2019 -Figura 1; durante el primer periodo, comprendido entre 1975 y 2000, la tasa anual de publicaciones permaneció estable, siendo la producción porcentual anual el 3,9\% (51). A partir del 2000, hubo un aumento del número de publicaciones en un $96 \%$ (1.241), lo cual determina un crecimiento del 92,1\%.

\section{Revistas}

El total de revistas fue de 425; 4 (20\%) de las 20 revistas con mayor número de publicaciones son de urología. - Figura 2. Sin embargo, si tomamos el total de las revistas, este porcentaje disminuye a un $2,64 \%$ (34). Las revistas con mayor porcentaje de publicaciones fueron: J Sex Med, (20,8\%; 270), J Sex Marital Ther (4,2\%; 55); Int J Impot Res (3,9\%; 51); Sex Med Rev (1,9\%; 25); y Sex Med (1,7\%; 22). Se encontraron 3 revistas con FI mayor a 8. La - Tabla 1 representa las revistas urológicas con publicaciones sobres el tema, su FI para el año 2018 según el Journal Citation Reports, la MCA, y la MCR.

\section{Autores}

Se encontraron 975 autores en Pubmed y, entre los 10 autores con mayor número de citaciones, las mujeres ocupan los 3 primeros puestos. Goldstein, I., fue el autor con mayor número de artículos sobre el tema investigado (32), con un índice h de 17 (-Tabla 2). 


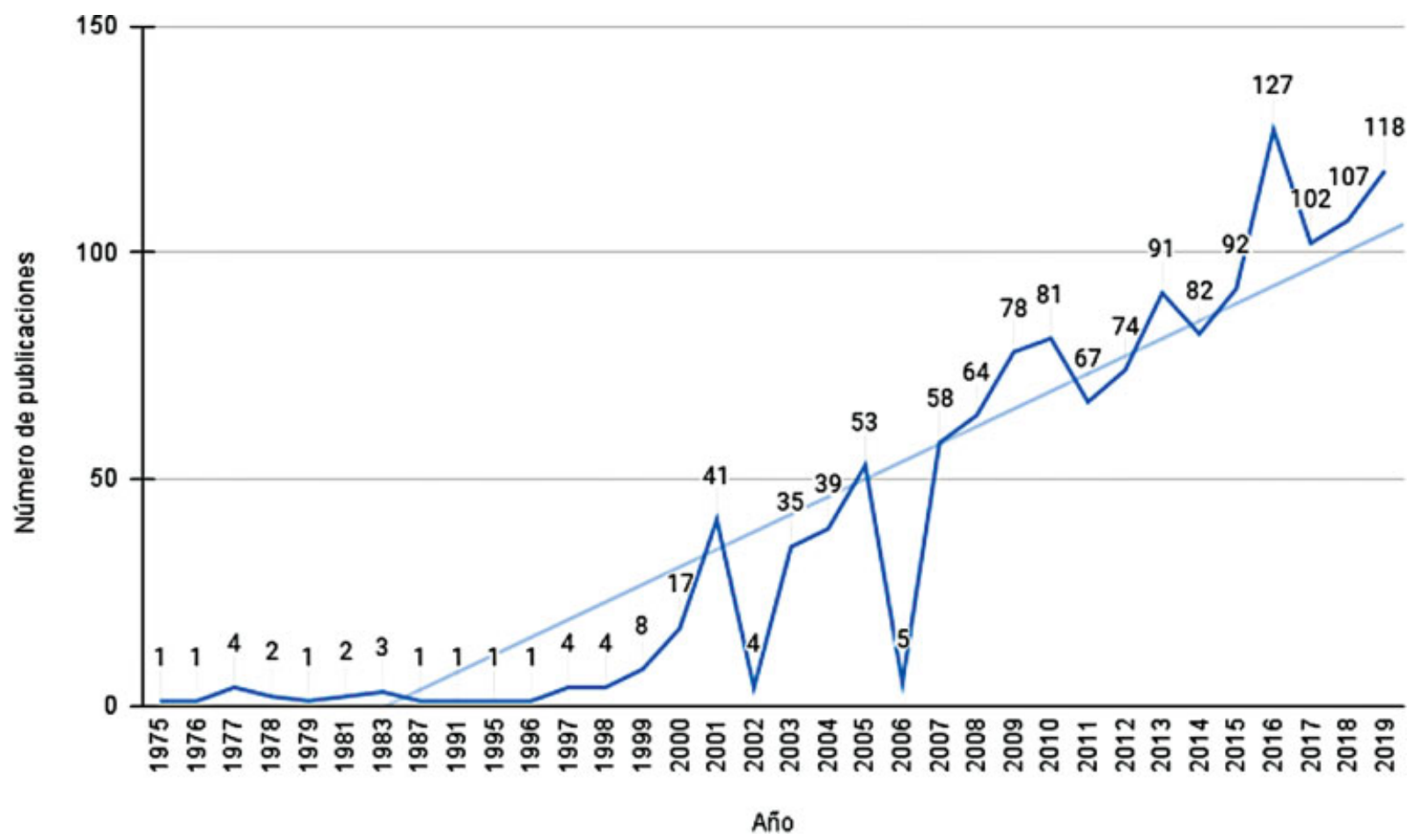

Fig. 1 Patrón de la producción científica del número de publicaciones por año sobre la disfunción sexual femenina durante el período 19752019.

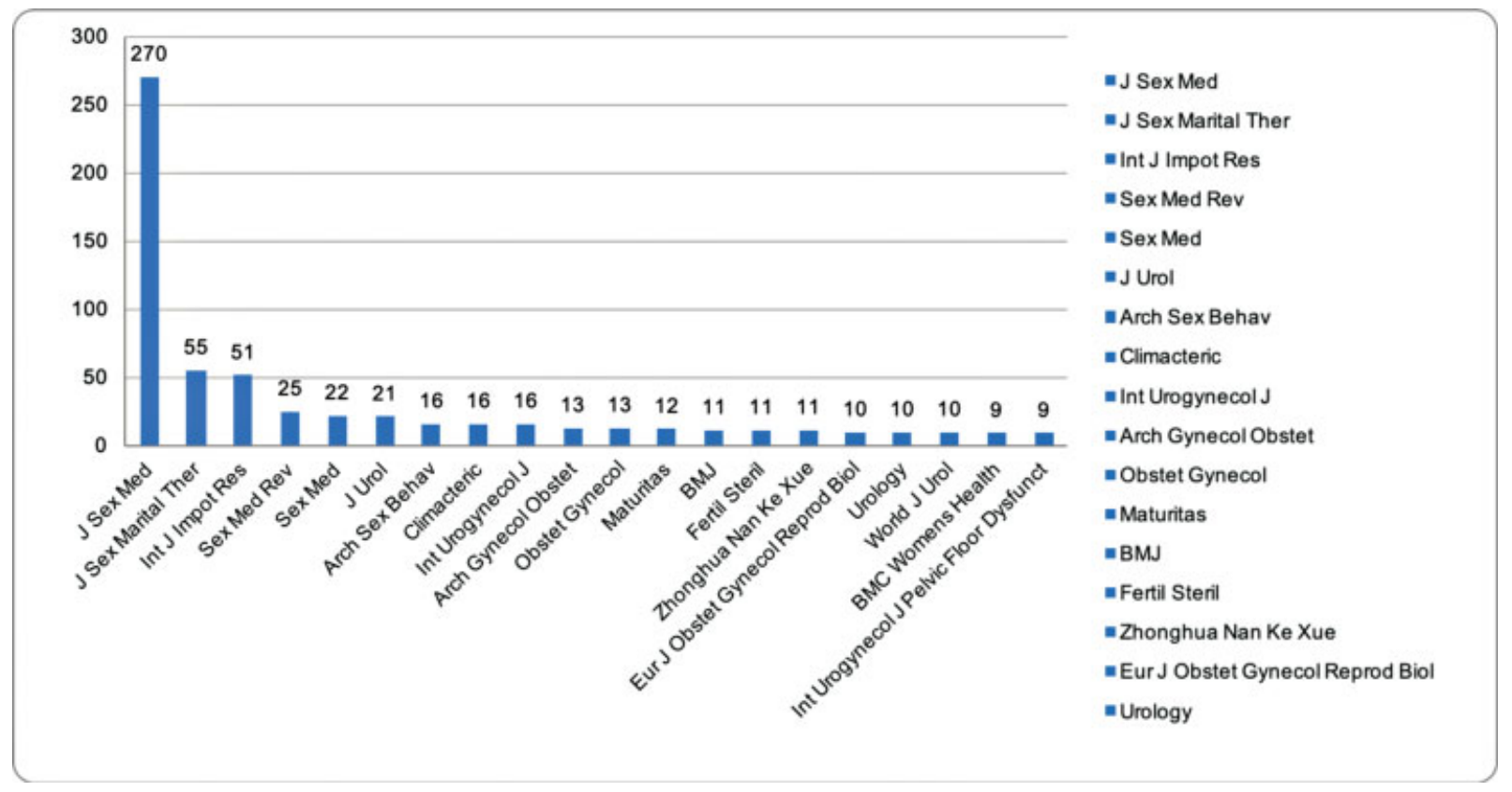

Fig. 2 Distribución de revistas con publicación sobre disfunción sexual femenina durante el período 1975-2019.

\section{Países}

La producción total de publicaciones se originó en 58 países. Los 10 principales países produjeron 1.011 artículos,con aproximadamente el $78,2 \%$ del total de publicaciones. Los primeros 10 países se clasificaron según el número total de publicaciones, y el mayor número provino de Estados Unidos $(31,4 \%)$ seguido de Reino Unido (12,9\%), Italia (8,9\%), Turquía (5,9\%), y Australia (4\%) - Figura 3. El país de América Latina con mayor número de publicaciones fue Brasil, (29), seguido de México (7), Colombia (3), y Argentina, Chile y Perú (2) - Figura 4.

\section{Artículos Más Citados}

Los 10 artículos con mayor número de citaciones se publicaron entre los años 2002 y 2010; solamente una publicación fue en una revista de urología. El artículo con 
Tabla 1 Listado de revistas de urología con publicación de artículos sobre disfunción sexual femenina de 1975 a 2019 , su factor de impacto para 2018, el número de publicaciones, el idioma, el país de publicación, el MCA, y el MCR

\begin{tabular}{|c|c|c|c|c|c|c|}
\hline Revista & Artículos & FI 2018 & Idioma & País & MCA & MCR \\
\hline J Urol & 21 & 5,64 & Inglés & Estados Unidos & 3 & 6 \\
\hline Int Urogynecol J & 16 & 2,09 & Inglés & Inglaterra & 1 & 2 \\
\hline Urology & 10 & 1,86 & Inglés & Estados Unidos & 1 & 2 \\
\hline World J Urol & 10 & 2,76 & Inglés & Estados Unidos & 2 & 3 \\
\hline Int Urogynecol J Pelvic Floor Dysfunct & 9 & 2,09 & Inglés & Inglaterra & $*$ & $F^{*}$ \\
\hline Curr Urol Rep & 8 & 2,79 & Inglés & Estados Unidos & 2 & 0 \\
\hline Prog Urol & 7 & 0,58 & Francés & Francia & 0 & 0 \\
\hline Curr Opin Urol & 6 & 2,05 & Inglés & Inglaterra & $\mathrm{n} / \mathrm{a}$ & $\mathrm{o}$ \\
\hline Eur Urol & 6 & 17,29 & Inglés & Países Bajos & 7 & 13 \\
\hline Urol Nurs & 6 & No tiene & Inglés & Inglaterra & * & * \\
\hline Int Urol Nephrol & 4 & 1,59 & Inglés & Países Bajos & 1 & 1.5 \\
\hline Neurourol Urodyn & 4 & 2,36 & Inglés & Estados Unidos & 1 & 2 \\
\hline Urologe A & 4 & $*$ & Alemán & Alemania & * & 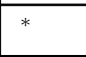 \\
\hline BMC Urol & 3 & 1,58 & Inglés & Inglaterra & 1 & 2 \\
\hline Turk J Urol & 3 & $*$ & * & * & $*$ & * \\
\hline Urol Clin North Am & 3 & 2,24 & Inglés & Estados Unidos & 1 & 6.5 \\
\hline Urol Int & 3 & 1,40 & Inglés & Suiza & 1 & 2 \\
\hline Urologiia & 3 & * & Ruso e inglés & Rusia & * & * \\
\hline Actas Urol Esp & 2 & 1,13 & Español & España & 0 & 1 \\
\hline Arab J Urol & 2 & $*$ & Inglés & Estados Unidos & * & $*$ \\
\hline Can Urol Assoc J & 2 & 1,25 & Inglés & Canadá & 1 & 1 \\
\hline Int J Urol & 2 & 2,10 & Inglés & Japón & 1 & 3 \\
\hline Korean J Urol & 2 & $*$ & Inglés & Corea & * & $*$ \\
\hline Nat Rev Urol & 2 & 9,33 & Inglés & Estados Unidos & $\overline{1}$ & 6 \\
\hline Transl Androl Urol & 2 & 2,11 & Inglés & China & 0 & 1 \\
\hline Aktuelle Urol & 1 & 0,31 & Multilengua & Alemania & 0 & 0 \\
\hline Arch Esp Urol & 1 & 0,33 & Multilengua & España & 0 & 0 \\
\hline Can J Urol & 1 & 1,03 & Inglés & Canadá & 0 & 2 \\
\hline Eur Urol Focus & 1 & $*$ & Inglés & Países bajos & 0 & 0 \\
\hline Int Braz J Urol & 1 & 1,04 & Inglés & Brasil & 1 & 1 \\
\hline J Pediatr Urol & 1 & 1,73 & Inglés & Inglaterra & 0 & 2 \\
\hline Nat Clin Pract Urol & 1 & * & Inglés & Inglaterra & 0 & 0 \\
\hline Rev Urol & 1 & 9,33 & Inglés & Estados Unidos & 1 & 6 \\
\hline Urol Res & 1 & $*$ & Inglés & Estados Unidos & 0 & 0 \\
\hline
\end{tabular}

Abreviaturas: Fl, factor de impacto; MCA, mediana de citas por artículo; MCR, mediana de citas por revisión.

mayor número de citaciones (417), fue «Definitions/ Epidemiology/Risk Factors for Sexual Dysfunction», de Lewis et al., publicado en el Journal of Sexual Medicine en el 2010 (-Tabla 3).

\section{Análisis por Agrupamiento}

Se realizó el análisis por agrupamiento y mapeo de los 1.292 artículos encontrados sobre disfunción sexual femenina, y se tomaron en cuenta las palabras clave y los títulos y resúmenes que tenían una correlación mínima de 5 veces. Esto dio como resultado 382 palabras, divididas en 9 grupos. Las palabras clave con mayor número de coocurrencias fueron: "female» (1.125), "humans» (1.119), y "female dysfunction, physiological» (689) (-Tabla 4). Tres de los nueve grupos concentran el mayor número de palabras. El grupo rojo (109 ítems) hace referencia a los tratamientos, y agrupa las palabras clave: "female», "treatment outcomes", «testosterone», y «hormone replacemente therapy»; el grupo 
Tabla 2 Listado de principales autores con más de 5 publicaciones sobre disfunción sexual femenina entre 1975 y 2019

\begin{tabular}{|l|l|l|l|}
\hline Autor & Artículos & Índice h & Institución y país \\
\hline Goldstein & 32 & 77 & Alvarado Hospital, San Diego, CA, EEUU \\
\hline Clayton & 28 & 43 & University of Virginia School of Medicine, Charlottesville, VA, EEUU \\
\hline Nappi & 25 & 52 & Fondazione IRCCS Policlinico San Matteo, Pavia, Italia \\
\hline Davis & 23 & 65 & Monash University, Melbourne, Australia \\
\hline Burri & 21 & 22 & European Institute for Sexual Health (EISH), Hamburgo, Alemania \\
\hline Esposito & 17 & 73 & Università degli Studi della Campania Luigi Vanvitelli, Nápoles, Italia \\
\hline Berman & 16 & 21 & David Geffen School of Medicine at UCLA, Los Angeles, CA, EEUU \\
\hline Rosen & 16 & 97 & New England Research Institutes, Watertown, MA, EEUU \\
\hline Derogatis & 15 & 54 & Maryland Center for Sexual Health, Lutherville-Timonium, MD, EEUU \\
\hline Jannini & 15 & 56 & Università degli Studi di Roma Tor Vergata, Roma, Italia \\
\hline
\end{tabular}

verde (70 ítems) hace referencia a la fisiopatología y síntomas, y agrupa la palabras clave: «incidence» y " quality of life»; y el grupo azul (53 ítems) hace referencia a la prevalencia, y tiene en cuenta las palabras clave: «adolescents», " young adult», y « risk factors» (- Figura 5).

\section{Discusión}

Las publicaciones sobre tópicos exclusivamente femeninos y, más aún, sobre sexualidad femenina no han sido muy

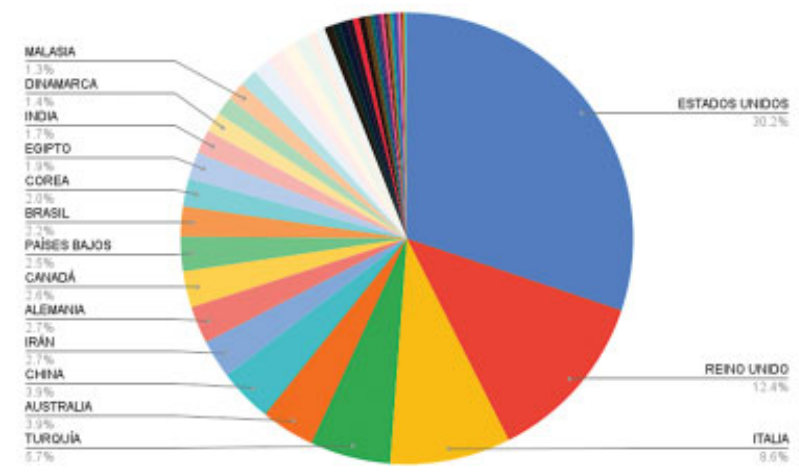

Fig. 3 Distribución porcentual de los países con publicación de artículos sobre disfunción sexual femenina durante el período 1975-2019.

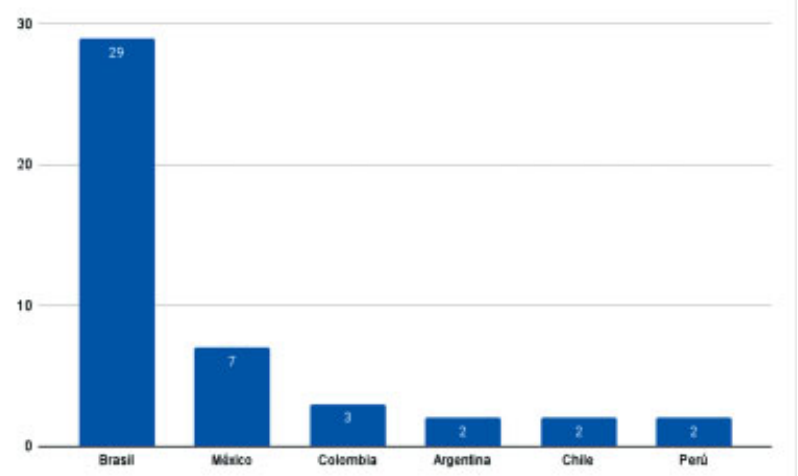

Fig. 4 Distribución de los países latinoamericanos con publicación de artículos sobre disfunción sexual femenina durante el período 1975-2019. frecuentes a lo largo de la historia. ${ }^{11}$ Es sólo después del año 2000 cuando comienzan a aparecer más publicaciones sobre este tópico, tras la aparición de terapias novedosas para disfunción eréctil, y tratando de buscar una clasificación que interpretara la problemática sexual en la mujer y posibles mecanismos para tratarla. ${ }^{12,13}$

Hubo muchos artículos en contra de estas publicaciones, tratando de interpretarlas como una invención de la industria farmacéutica en un intento por abarcar un nuevo mercado que estaba desatendido y lograr un boom como el del Viagra. Sin embargo, con el pasar de los años, se ha evidenciado que las disfunciones sexuales femeninas sí existen, y debe buscarse activamente su seguimiento y tratamiento. ${ }^{14}$ Esto se corrobora en los hallazgos de este estudio, en el que se encontró desde 1975 hasta el año 2000, una estabilidad en la tasa anual de publicación de artículos sobre disfunción sexual femenina (3,72\%; 51), y, de allí en adelante, un aumento exponencial de dicha producción, determinando un crecimiento del 92,55\% con un total de 425 revistas, de las cuales solamente 34 corresponden a esta especialidad (2,69\%). Haciendo un análisis detallado sobre las palabras clave en las búsquedas, encontramos que las que más aparecen son "female», con 1.125, "humans», con 1.119, lo cual no es de extrañar, seguidas por "female dysfunction" y "physiological» con 684 . Al realizar la evaluación de estos artículos, vemos que la mayoría abarca temas sobre tratamiento, resultados de tratamiento, reemplazo hormonal $\mathrm{y}$ tratamiento, $\mathrm{y}$, en segundo lugar, temas de fisiopatología y síntomas; esto nos permite identificar que es un campo en que activamente se continúa estudiando su origen, fisiopatología, posibles clasificaciones $\mathrm{y}$, sobretodo, en que aun hay mucho que escribir sobre tratamientos y posibles desenlaces.

Las revistas con mayor porcentaje de publicaciones fueron: $J$ Sex Med (19,72\%; 270), J Sex Marital Ther (4,01\%; 55), Int J Impot $\operatorname{Res}(3,72 \% ; 51)$, Sex Med $\operatorname{Rev}(1,82 \% ; 25)$, y Sex $\operatorname{Med}(1,60 \% ; 22)$. Con respecto a las publicaciones urológicas sobre el tema, también se identifica un ascenso paulatino, siendo el Journal Urology el con el mayor número de publicaciones, y el European Urology, el con el mayor FI $(17,29)$. De los 10 artículos mas citados, todos publicados desde 2002, solo 1 revista - European Urology-es netamente urológica, y hace referencia a la DSFen el 
Tabla 3 Listado de los diez artículos más citados

\begin{tabular}{|c|c|c|c|c|c|}
\hline Rango & Título & Autores & Citas & Revista & $\begin{array}{l}\text { Año de } \\
\text { publicación }\end{array}$ \\
\hline 1 & $\begin{array}{l}\text { Definitions/Epidemiology/Risk } \\
\text { Factors for Sexual Dysfunction }\end{array}$ & Lewis et al. & 417 & Journal of Sexual Medicine & 2010 \\
\hline 2 & $\begin{array}{l}\text { The female sexual distress scale } \\
\text { (FSDS): Initial Validation of a } \\
\text { Standardized Scale for Assessment of } \\
\text { Sexually Related Personal Distress in } \\
\text { Women }\end{array}$ & Derogatis et al. & 403 & Journal of Sex \& Marital Therapy & 2002 \\
\hline 3 & $\begin{array}{l}\text { Epidemiology/Risk Factors of Sexual } \\
\text { Dysfunction }\end{array}$ & Lewis et al. & 340 & Journal of Sexual Medicine & 2004 \\
\hline 4 & $\begin{array}{l}\text { Prevalence of Male and Female Sexual } \\
\text { Dysfunction Is High } \\
\text { Following Surgery for Rectal Cancer }\end{array}$ & Hendren et al. & 336 & Annals of Surgery & 2005 \\
\hline 5 & $\begin{array}{l}\text { Female Androgen Insufficiency: the } \\
\text { Princeton Consensus Statement on } \\
\text { Definition, Classification, and } \\
\text { Assessment }\end{array}$ & Bachmann et al. & 281 & Fertility and Sterility & 2002 \\
\hline 6 & $\begin{array}{l}\text { Validation of the Female Sexual } \\
\text { Distress Scale-Revised for Assessing } \\
\text { Distress in Women with Hypoactive } \\
\text { Sexual Desire Disorder }\end{array}$ & DeRogatis et al. & 271 & Journal of Sexual Medicine & 2008 \\
\hline 7 & $\begin{array}{l}\text { The Making of a Disease: Female } \\
\text { Sexual Dysfunction }\end{array}$ & Moynihan & 218 & British Medical Journal & 2003 \\
\hline 8 & EAU Guidelines on Chronic Pelvic Pain & Fall et al. & 215 & European Urology & 2010 \\
\hline 9 & $\begin{array}{l}\text { What Is the "True" Prevalence of } \\
\text { Female Sexual Dysfunctions and } \\
\text { Does the Way We Assess These } \\
\text { Conditions Have an Impact? }\end{array}$ & Hayes et al. & 200 & Journal of Sexual Medicine & 2008 \\
\hline 10 & $\begin{array}{l}\text { Risk Factors for Female Sexual } \\
\text { Dysfunction in the General } \\
\text { Population: Exploring Factors } \\
\text { Associated with Low Sexual } \\
\text { Function and Sexual Distress }\end{array}$ & Hayes et al. & 195 & Journal of Sexual Medicine & 2008 \\
\hline
\end{tabular}

Tabla 4 Palabras clave con mayor número de coocurrencias en publicaciones sobre disfunción sexual femenina

\begin{tabular}{|l|l|}
\hline Palabras clave & Ocurrencias \\
\hline Female & 1.125 \\
\hline Humans & 1.119 \\
\hline Sexual dysfunction, physiological & 684 \\
\hline Adult & 563 \\
\hline Sexual dysfunction, psychological & 627 \\
\hline Middle-aged & 462 \\
\hline Surveys and questionnaires & 332 \\
\hline Sexual behavior & 278 \\
\hline Young adult & 175 \\
\hline Aged & 171 \\
\hline Male & 217 \\
\hline
\end{tabular}

Tabla 4 (Continued)

\begin{tabular}{|l|l|}
\hline Palabras clave & Ocurrencias \\
\hline Cross-sectional studies & 164 \\
\hline Prevalence & 166 \\
\hline Risk factors & 152 \\
\hline Quality of life & 141 \\
\hline Libido & 144 \\
\hline Female sexual dysfunction & 183 \\
\hline Orgasm & 116 \\
\hline Adolescent & 106 \\
\hline Women's health & 106 \\
\hline Sexuality & 107 \\
\hline Treatment outcome & 94 \\
\hline
\end{tabular}




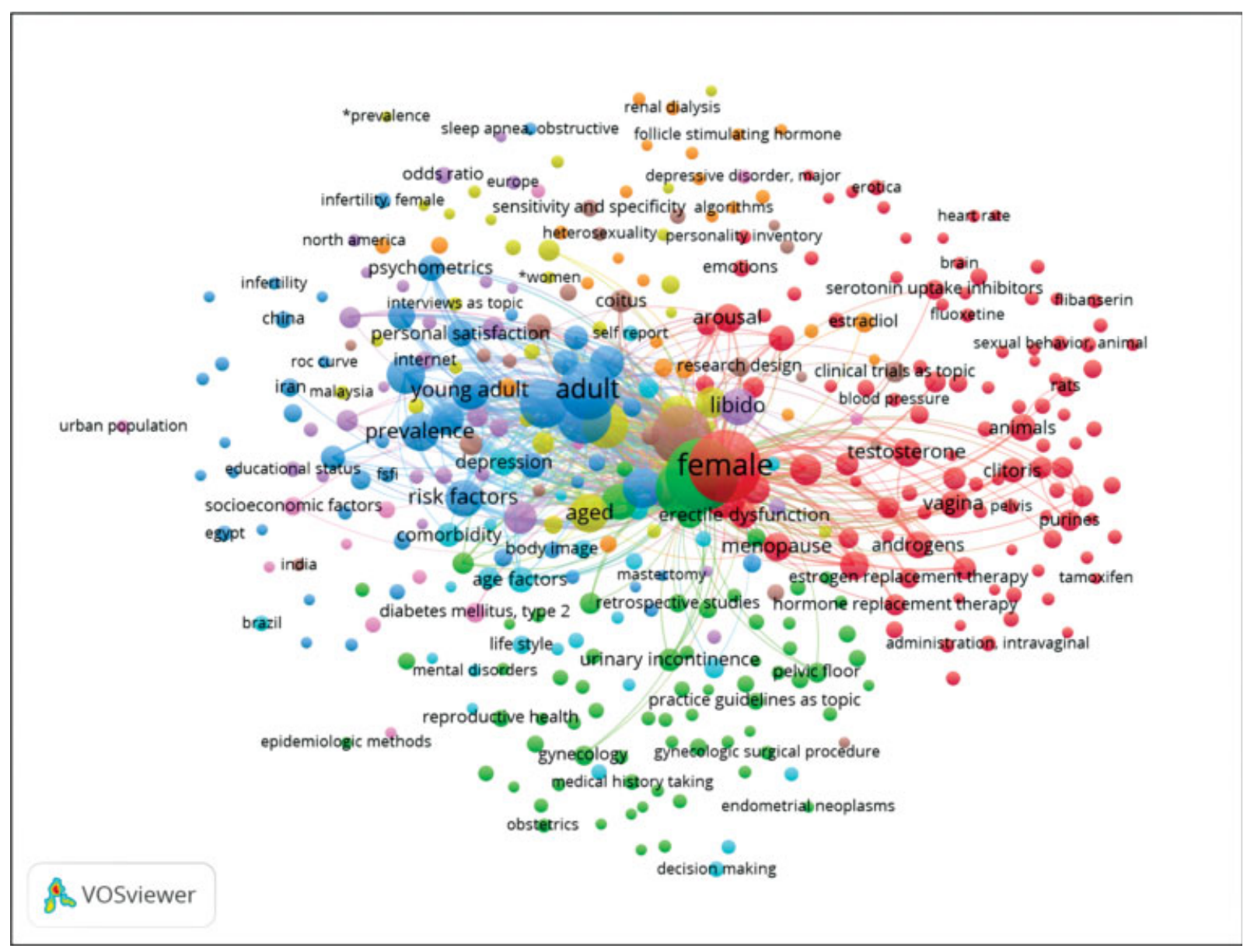

Fig. 5 Análisis y mapeo de palabras clave con mayor número de coocurrencia divididas en grupos.

contexto del dolor pélvico crónico. El ascenso en la producción de artículos sobre sexualidad femenina contrasta con el mínimo entrenamiento sobre el tema durante los programas de residencia. ${ }^{13}$ Estos hallazgos fueron encontrados por Pauls et al. $^{16}$ en encuestas realizadas entre miembros de la American Urogynecologic Society (AUGS), y la British Society of Urogynecology (BSUG) mostró que la minoría (22\%) de sus miembros evaluó a todos sus pacientes para detectar DSF. ${ }^{15}$

Por consiguiente, no se encuentran en la literatura análisis bibliométricos de la DSF, lo que podría explicarse debido a las variaciones en las definiciones, las diferentes categorías de diagnóstico utilizadas, la muestra de las publicaciones, y los métodos de recolección de datos. ${ }^{17,18}$ Tomamos como referencia publicaciones similares en diferentes tópicos que puedan darnos parámetros de comparación, como el estudio de Rezaee et al. ${ }^{19}$ (2018), sobre disfunción eréctil, en que se encontró un $34,4 \%$ de crecimiento de número de autores por manuscrito, un 38,9\% $(p<0,001)$ de incremento de artículos originales sobre el tema, y un 36,5\% de aumento en artículos de revisión.

En el presente estudio, encontramos un porcentaje considerable de mujeres (aunque de otras especialidades), que escriben sobre el tema, que se ubican en los cuatro primeros lugares en publicaciones sobre DSF, así como en otras especialidades, con estudios similares. ${ }^{20,21}$ De los 975 autores encontrados en nuestra revisión, 18 tuvieron más de
5 publicaciones, y la autora con mayor número de publicaciones fue Clayton, $\mathrm{AH}$.

Adicionalmente, la participación femenina en artículos sobre DSF en revistas de alto FI viene incrementándose desde hace un par de décadas, pasando de un tema tabú ${ }^{22}$ hasta el escenario actual, en el cual vemos el liderazgo femenino al abordar la problemática. ${ }^{23} \mathrm{Si}$ bien es cierto que en otros temas de diferentes especialidades aún falta mucho para lograr liderazgo, ${ }^{24}$ en disfunciones sexuales femeninas vamos por buen camino: en 1.298 artículos relacionados con el tema, de los 10 autores principales, 8 son mujeres, pero ninguna de la especialidad de urología.

El mayor número de publicaciones pertenece a Estados Unidos (29,6\% (406) seguido de Reino Unido (12.19\%; 167); esto no difiere mucho a otros temas en la literatura urológica: entre los 10 principales países, se produjeron 1.011 artículos, que comprenden aproximadamente el $73.8 \%$ del total de publicaciones. El país latinoamericano que más escribe es Brasil (29 publicaciones), seguido por México (7), y Colombia (3), lo cual es un pequeño porcentaje si lo comparamos con los de otros países.

Entre las limitaciones, se encuentra la barrera del idioma, pues la mayoría de las publicaciones son en inglés, y, aún sin limitar el idioma, en nuestra búsqueda puede existir literatura que no se haya incluido para este estudio. Los motores de búsqueda, como PubMed, sólo incluyen la 
dirección del primer autor, por lo que no se puede determinar la cantidad de artículos de colaboración multinacional, y esto a su vez reduciría la posibilidad de tener un cálculo confiable de la producción en países en desarrollo. Adicionalmente, en las bases de datos consultadas, pueden existir artículos en la búsqueda que cubren otro tipo de tópicos.

A pesar de las limitaciones, la metodología de búsqueda no sólo puede reproducirse, sino que ya ha sido utilizada por otros autores, ${ }^{25-27}$ por lo que nuestros resultados pueden ser comparados con futuros manuscritos.

\section{Conclusión}

Este análisis bibliométrico mostró las diferentes tendencias y tasas de publicación de estudios sobre DSF, y evidenció una baja tasa de producción con relación a otras temáticas, sobre todo en las revistas urológicas, con predominio de mujeres como autores, aunque en los últimos años se ha identificado un crecimiento importante y sostenido. Se hace evidente la necesidad de desarrollar estrategias para mejorar la formación tanto en los niveles de posgrado como de pregrado, y así generar más adhesión a este tema en una especialidad quirúrgica como la nuestra.

\section{Conflicto de Intereses}

Las autoras no tienen conflicto de intereses que declarar.

\section{Referencias}

1 Basson R. The Female Sexual Response Revisited. J SOGC 2000;22 (05):378-382. Doi: 10.1016/S0849-5831

2 Laan E, Everaerd W, van der Velde J, Geer JH. Determinants of subjective experience of sexual arousal in women: feedback from genital arousal and erotic stimulus content. Psychophysiology 1995;32(05):444-451. Doi: 10.1111/j.1469-8986.1995.tb02095.x

3 Bancroft J. Sexual Desire and the Brain. Sex Marital Ther 1998;3 (01):11-27. Doi: 10.1080/02674658808407689

4 Laumann EO, Paik A, Rosen RC. Sexual dysfunction in the United States: prevalence and predictors. JAMA 1999;281(06):537-544. Doi: $10.1001 /$ jama.281.6.537

5 Alcántara Montero A, Sánchez Carnerero CI. Disfunción sexual femenina: opciones de tratamiento farmacológico. Semergen 2016;42(05):e33-e37. Doi: 10.1016/j.semerg.2016.02.008

$6 \mathrm{NIH}$ Consensus Development Panel on Impotence. NIH Consensus Conference. Impotence. JAMA 1993;270(01):83-90. Doi: 10.1001/ jama.270.1.83

7 Rosen RThe Process of Care Consensus Panel. The process of care model for evaluation and treatment of erectile dysfunction. Int J Impot Res 1999;11(02):59-70, discussion 70-74. Doi: 10.1038/sj. ijir.3900411

8 Rosen RC. Sexual function assessment and the role of vasoactive drugs in female sexual dysfunction. Arch Sex Behav 2002;31(05): 439-443. Doi: 10.1023/A:1019896226072

9 Tiefer L. A New View of Women's Sexual Problems: Why New? Why Now?. J Sex Res 2001;38(02):89-96. Doi: 10.1080/ 00224490109552075

10 Ellegaard O, Wallin JA. The bibliometric analysis of scholarly production: How great is the impact? Scientometrics 2015;105 (03):1809-1831. Doi: 10.1007/s11192-015-1645-z

11 Castaño-López E, Plazaola-Castaño J, Bolívar-Muñoz J, Ruiz-Pérez I, (n.d.) Publicaciones sobre mujeres, salud y género en España
(1990-2005). Rev. Esp. Salud Publica80(6), 705-716 Retrieved May 27, 2020, from 10.1590/s1135-57272006000600010

12 Basson R, Berman J, Burnett A, et al. Report of the international consensus development conference on female sexual dysfunction: definitions and classifications. J Urol 2000;163 (03):888-893. Doi: 10.1016/s0022-5347(05)67828-7

13 Gabbard GO. Musings on the report of the International Consensus Development Conference on Female Sexual Dysfunction: definitions and classifications. J Sex Marital Ther 2001;27(02):145-147 Retrieved May272020. Doi: 10.1080/ 00926230152051824

14 Dalpiaz O, Kerschbaumer A, Mitterberger M, et al. Female sexual dysfunction: a new urogynaecological research field. BJU Int 2008;101(06):717-721 Retrieved May272020 . Doi: 10.1111/ j.1464-410x.2007.07442.x

15 Milou B, Beck J. The Place of Female Sexual Dysfunction in the Urological Practice: Results of a Dutch Surveyjsm_1460.J Sex Med 2009;(06):2979-2987https://pubmed.ncbi.nlm.nih.gov/ $19694924 /$

16 Pauls RN, Kleeman SD, Segal JL, Silva WA, Goldenhar LM, Karram MM. Practice patterns of physician members of the American Urogynecologic Society regarding female sexual dysfunction: results of a national survey. Int Urogynecol J Pelvic Floor Dysfunct 2005;16(06):460-467

17 Clayton AH, Valladares Juarez EM. Female Sexual Dysfunction. Psychiatr Clin North Am 2017;40(02):267-284

18 Hayes RD, Dennerstein L, Bennett CM, Fairley CK. What is the "true" prevalence of female sexual dysfunctions and does the way we assess these conditions have an impact? J Sex Med 2008;5(04): 777-787

19 Rezaee ME, Johnson HA, Munarriz RM, Gross MS. Bibliometric Analysis of Erectile Dysfunction Publications in Urology and Sexual Medicine Journals. J Sex Med 2018; 15(10):1426-1433

20 Xiao N, Oliveira DFM, Gupta R. Characterizing the Impact of Women in Academic IR: A 12-Year Analysis. J Vasc Interv Radiol 2018;29(11):1553-1557 Retrieved May272020 . Doi: 10.1016/j. jvir.2018.06.010

21 Campbell JC, Yoon SC, Grimm LJ. Collaboration Metrics Among Female and Male Researchers: A 5-Year Review of Publications in Major Radiology Journals. Acad Radiol 2018;25(07):951-954 Retrieved May272020 . Doi: 10.1016/j.acra.2017.12.034

22 O'Rand AM. Women in Science: Career Processes and Outcomes. By Yu Xie and Kimberlee A. Shauman. Harvard University, 2003. 318 pp. Social ForcesVolume 82, Issue 4, June 2004, Pages 1669 -1671. Doi: $10.1353 /$ sof.2004.0086

23 Giner-Soriano M, López-Pereiro O, Zabaleta-del-Olmo E, PonsVigués M, Morros R, Gómez-Lumbreras A. Bibliometric analysis of female authorship in original articles in the journal ATENCIóN PRIMARIA. Aten Primaria 2021;53(01):12-18

24 Silver JK, Poorman JA, Reilly JM, Spector ND, Goldstein R, Zafonte RD. Assessment of Women Physicians Among Authors of Perspective-Type Articles Published in High-Impact Pediatric Journals. JAMA Netw Open 2018;1(03):e180802 Retrieved May272020 . Doi: 10.1001/jamanetworkopen.2018.0802

25 Sugimoto CR, Ahn YY, Smith E, Macaluso B, Larivière V. Factors affecting sex-related reporting in medical research: a crossdisciplinary bibliolátrico analysis. The Lancet 2019;393(10171): 550-559

26 Xiao N, Mansukhani NA, Oliveira DFMd, Kibbe MR. Association of author gender with sex bias in surgical research. JAMA Surgery 2018;153(07):663-670

27 Ouyang D, Sing D, Shah S, Hu J, Duvernoy C, Harrington RA, Rodriguez F. Sex disparities in authorship order of cardiology scientific publications: trends over 40 years. Circulation: Cardiovascular Quality and Outcomes 2018;11(12):e005040 